\title{
Cinéma féministe argentin : les questionnements épistémologiques et politiques d'Albertina Carri
}

\section{Michèle Soriano}

\section{(2) OpenEdition \\ Journals}

Édition électronique

URL : https://journals.openedition.org/ges/2133

DOI : $10.4000 /$ ges. 2133

ISSN : 2431-6563

\section{Éditeur}

Presses universitaires de Bordeaux

\section{Édition imprimée}

Date de publication : 1 janvier 2016

Pagination : 131-156

\section{Référence électronique}

Michèle Soriano, «Cinéma féministe argentin : les questionnements épistémologiques et politiques d'Albertina Carri », Genre en séries [En ligne], 3 | 2016, mis en ligne le 07 février 2022, consulté le 10 février 2022. URL : http://journals.openedition.org/ges/2133; DOI : https://doi.org/10.4000/ges.2133

\section{(9) $(\mathcal{\Theta \Theta}$}

La revue Genre en séries est mise à disposition selon les termes de la Licence Creative Commons Attribution - Pas d'Utilisation Commerciale - Pas de Modification 4.0 International. 


\title{
CINÉMA FÉMINISTE ARGENTIN : LES QUESTIONNEMENTS ÉPISTÉMOLOGIQUES ET POLITIQUES D'ALBERTINA CARRI
}

\author{
Michèle SORIANO
}

\section{RÉSUMÉ}

À partir de ce que la cinéaste argentine Albertina Carri nomme les «excès de normalité » et de ses déconstructions de la violence de genre, je voudrais soulever quelques questions à propos du féminisme dans l'art, des stratégies de normalisation du discours féministe et des ruptures du sens commun que celui-ci promeut. Je souhaite exposer la position radicale qu'adopte Albertina Carri, qui associe sans concession l'invention formelle au questionnement des catégories, et montrer que cet avant-gardisme qu'elle revendique, s'il pose le problème de l'accessibilité de sa production cinématographique, sans cesse hors normes parce qu'elle se positionne sans trêve en tant qu'autre disloquée (Maria Luisa Femenías), est à la fois l'expression d'un questionnement épistémologique et politique féministe issu des pratiques de luttes populaires en Argentine.

MOTS-CLÉS: ALBERTINA CARRI, DISGOURS FÉMINISTE ET QUEER, CINÉMA ARGENTIN, SÉRIES TV, DROITS À L'IDENTITÉ

\section{ABSTRACT}

Based on what Albertina Carri, an Argentine filmmaker, calls the « excess of normality » and on her deconstruction of gender violence, I would like to raise some issues about feminism in art. I will focus on the strategies aiming at normalizing feminist discourses as well as the failure of common sense that it promotes. I want to expose the radical position adopted by Albertina Carri, who combines uncompromising formal invention and the questioning of categories. I'll try to show that her vanguard thinking, even though it raises the issue of the accessibility of her production due to her positioning as another dislocada (Maria Luisa Femenías), is an expression of epistemological and political feminist questioning derived from popular grassroots movements in Argentina.

KEYWORDS : ALBERTINA CARRI, FEMINIST AND QUEER DISCOURSE, ARGENTINIAN CINEMA, TV SERIES, RIGHT TO IDENTITY 
Michèle Soriano (PhD Études latino-américaines, Université de Pittsburgh) est professeure à l'Université Toulouse - Fean Faurès. Spécialiste des études de genre dans la culture hispanoaméricaine, elle dirige un groupe de recherche au sein de l'Institut de Recherches Intersites Etudes Culturelles (IRIEC - Toulouse) qui se consacre aux études de genre dans la littérature, le cinéma et les sociétés hispano-américaines. Ses publications portent essentiellement sur les écrivaines et les cinéastes latino-américaines actuelles; elle a dirigé ou co-dirigé plusieurs dossiers de revue et livres collectifs dont les récents Caravelle $n^{\circ} 102$ "Citoyenneté et formes de violence. La violence de genre en Amérique latine» (PUM, 2014) avec Maria Luisa Femenías, et, avec Laurence Mullaly, De cierta manera : cine y género en América latina, (Paris, L'Harmattan, 2014). Depuis 2005 elle coordonne, avec Mónica Zapata, le séminaire interuniversitaire Toulouse - Tours: "Lectures du genre dans la production culturelle espagnole et latino-américaine» et participe aux travaux du réseau Genre pluridisciplinaire ARPĖGE de la MSHS - Toulouse. 
En realidad, hay un momento en que sufro que es cuando empiezan a aparecer signos de «exceso de normalidad» (Albertina Carri $)^{1}$.

Albertina Carri, cinéaste argentine, commente en ces termes, dans une interview (Iribarren, 2008), la fonction des séquences d'animation dans son dernier long métrage de fiction intitulé La Rabia/La Rage (2008). Ce film met en scène deux enfants hors normes, l'un boiteux, l'autre autiste, en tant que figures de résistance à la violence naturalisée qui structure le microcosme rural contemporain dans lequel ils grandissent (Mullaly, 2014). L'évocation de cette violence «excessivement normale» qu'identifie Albertina Carri me servira, dans cette brève réflexion, à poser quelques questions à propos du féminisme dans l'art, des stratégies de normalisation du discours féministe et des ruptures du sens commun que celui-ci promeut.

Ces propos de Carri m'ont rappelé une anecdote que je rapporterai ici pour l'enseignement que j’ai pu y puiser. En 1990 j’ai assisté à San Cristóbal de las Casas à un hommage à une grande écrivaine mexicaine, Rosario Castellanos (1925-1974), qui est l'une des figures majeures de l'indigénisme et du féminisme mexicain. Lors d'une table ronde, des écrivains qui furent ses compagnons de route témoignèrent de son parcours. L’un d'eux commença son discours en précisant: «Rosario Castellanos n'était pas féministe, c'était une femme normale ». Vingt-cinq ans plus tard, je m'interroge encore au sujet de cette affirmation, aussi absurde qu'injurieuse, pour les féministes, mais surtout pour l'œuvre et l'engagement de Castellanos ${ }^{2}$. Je mesurais après coup, avec amertume et colère, comment

1 «En réalité, il y a un moment où je me mets à souffrir, c'est quand commencent à apparaître des signes 'd'excès de normalité' » (Iribarren, 2008, ma traduction).

2 J'étais en compagnie d'une spécialiste de l'œuvre de Castellanos, Aralia López; nous échangeâmes un regard complice et un rire embarrassé ; je déclarais tout bas : « Alors nous, les féministes, sommes donc des femmes subnormales! » Notre silence m’a longtemps pesé, avant que je puisse comprendre l'effet de sidération que peuvent avoir ces violences verbales ainsi que le blocage cognitif que leur répétition incessante produit, un «verrouillage » auquel les normes du discours savant et ses modes de constitution institutionnalisés, comme le montre Michèle Le Dœuff dans Le Sexe du savoir (1998), ne sont pas étrangers. Si cette 
cet «excès de normalité » était destiné, paradoxalement, à conforter la canonisation de l'écrivaine, au prix du sens et de la valeur de sa production. Je reconsidère maintenant l'anecdote à la lumière de la lecture des propos de Françoise Collin, qui commente le postulat qu'elle a un jour formulé ainsi : « tout art de femme est féministe »(Collin et Kaufer, 2014 : 135-157).

Collin mène une intéressante réflexion à propos de la double contrainte qui s'exerce sur le discours des femmes - ces mécanismes d'exclusion de l'autorité discursive que Michèle Le Dœuff a nommés la « nomophatique » dans son essai intitulé Le Sexe du savoir (Le Dœuff, 1998 : 116). Collin évoque les différentes stratégies qui peuvent être adoptées pour en déjouer les effets. Le travail de la critique féministe - tel que le définit Collin - serait de : «relayer et donner corps à l'œuvre d'une femme. Et le faire sans la retenir pour autant dans le seul cercle des femmes, ce qui serait la reconditionner: lui donner corps sans l'approprier, la révéler comme œuvre à signification universalisable » (Collin et Kaufer, 2014 : 141). Il me paraît intéressant d'assumer le postulat de Collin : tout art de femme est probablement, d'une certaine façon, féministe, mais - pouvons-nous nous demander - convient-il, pour le faire exister, pour contribuer à le faire reconnaître en tant qu'art, pour en assurer la diffusion, d'en nier, effacer ou minimiser la portée féministe?

Nous abordons ici une question que nous ne pourrons qu'évoquer superficiellement, qui concerne l'art, mais également la critique, le champ culturel et le champ du savoir (Naudier et Rollet, 2007 ; Sofio, Yavuz et Molinier, 2007): la question des rapports entre le discours féministe et l'universalité, celle de l'universalisation des savoirs. La construction de l'autorité d'une position d'énonciation requiert-elle l'effacement des rapports

anecdote peut paraître déplacée dans un tel travail, mon hypothèse cependant s'inspire du patient travail que réalise Le Dœuff dans son essai, qui met en rapport, comme issus d'un cadre commun, certains traits des «savoirs institués » et de la «vie au jour le jour » (Le Dœuff, 1998 : 17). Suivant cette hypothèse, que j’ai exposée ailleurs (Soriano, 2014b), le malaise ressenti lors de cet événement - partagé par Aralia López - peut-être ressaisi en tant que déploiement d'une deuxième scène d'énonciation, interdite ou invisibilisée par la violence de genre, en tant que continuum auquel l'institution universitaire n'échappe pas ; la possibilité d'en rendre compte et d'y prendre part, en restituant la parole mise en suspens, doit par conséquent, d'une certaine façon, franchir certaines bornes qui définissent le cadre discursif universitaire. Sur la vertu cognitive de la non-conformité, on lira la synthèse de Ludovic Gaussot (2005). 
sociaux de sexe qui la définissent et, dans le cas qui nous intéresse, la désautorisent? Quel est le coût de l'universalisme tel que nous le pratiquons? Quelles sont les conséquences de la nomophatique dans l'art? Et de ce «blocage cognitif » (Le Dœuff, 1998 : 17) qu'engendre ce que Carri identifie comme des « excès de normalité »?

Ce que Collin met en lumière en tant que féminisme dans l'art des femmes, ce sont les inventions de nouvelles formes, la production de techniques et de savoirs qui vont contribuer à façonner l'imaginaire commun (Collin et Kaufer, 2014: 144). Cette donnée fait partie des discours sur l'art et est universalisable. Collin souligne cependant l'accès difficile à ces œuvres qui «innovent», car «la grille de lecture traditionnelle » qu'on leur applique dissimule - dit-elle - leur «caractère générateur » (Collin et Kaufer, 2014 : 138). Or ce sont ces grilles de lecture et leur fonctionnalité dans le cadre herméneutique qui les légitime, qui vont déterminer la reconnaissance des travaux critiques et donc des œuvres ciblées : toute une entreprise de normalisation du discours artistique, jusque dans le type de ruptures qu'il opère, est en jeu dans le discours critique, qui tend à effacer certains des questionnements engendrés ou à les déplacer vers des problématiques canoniques.

L'une des tâches de la critique féministe pourrait être de reconstruire l'archive invisible du discours féministe dans l'art: autrement dit, non seulement de faire exister les productions artistiques des femmes dans l'histoire de l'art, en mettant en lumière l'universalité de leurs œuvres, mais également d'universaliser les questionnements épistémologiques et politiques que leurs productions portent.

Dans le cadre de cette démarche, je souhaite aujourd'hui exposer la position radicale qu'adopte Albertina Carri (Lopez Riera, 2009), dans la mesure où elle associe sans concession l'invention formelle au questionnement des catégories. Cet avant-gardisme qu'elle revendique pose le problème de l'accessibilité de sa production cinématographique, sans cesse hors normes, parce qu'elle se positionne sans trêve en tant qu'autre disloquée - pour reprendre l'expression de la philosophe argentine Maria Luisa Femenías - c'est-à-dire qu'elle est de celles et ceux qui « refusent d'occuper 
(et d'adopter) la forme et le lieu que les récits hégémoniques leur confèrent » (Femenías, 2006 : 101 - ma traduction).

\section{GARRI, ANNÉES 2000}

Avant d'étudier deux productions télévisuelles réalisées par Carri, un téléfilm sur la question de la dépénalisation de l'avortement et une série télé sur les droits à l'identité, il est nécessaire d'exposer et de contextualiser les prises de position éthiques, politiques et esthétiques de la cinéaste. Une de ses interventions nous aidera à les synthétiser.

Lors de la conférence qu'elle a donnée dans un colloque que nous avons organisé à Toulouse en 2012, Albertina Carri opposait le fonctionnement de la télévision et celui du cinéma, et proposait de réviser certaines catégories. Elle définissait de cette façon sa position en tant que cinéaste :

Vous allez me dire qu'il existe un cinéma commercial ou industriel, un cinéma qui se complaît avec ses propres moyens à imposer une pensée hégémonique et hétérosexiste. Le mélodrame serait le paradigme de cette rhétorique. Toutefois, ce qui est véritablement cinématographique présente toujours des failles, travaille toujours sur un état de la diégèse, à la différence de la télévision qui fonde tout son discours sur une fausse mimesis. C'est pour cela que nous considérons simplement que cette technologie que l'on appelle cinéma industriel est en réalité un engrenage de plus dans le système des mass media.

Une fois dit cela, je propose, à partir de ce forum, que nous éradiquions de nos langues les catégories de cinéma d'auteur, cinéma de masse, cinéma industriel, cinéma commercial, cinéma indépendant, cinéma d'art, cinéma de divertissement et que nous appelions simplement cinéma tous ces films capables de transcender l'illusion du mouvement à l'écran en interpellant les spectateurs sur leur réalité, en proposant de nouvelles réalités, en s'opposant aux pouvoirs qui oppriment. Nous appellerons tout le reste propagande (Carri 2013).

Il convient de dessiner à grands traits le contexte d'énonciation de la réalisatrice afin de comprendre le sens de ces propos. Albertina Carri est la fille de Ana María Caruso et de Roberto Carri, intellectuels engagés, cadres dans le mouvement révolutionnaire de guérilla urbaine Montoneros, enlevés, séquestrés par la Junte militaire en 1977, torturés puis assassinés un an plus 
tard. Carri, née en 1973, n’a que 4 ans quand ses parents « disparaissent ». Elle est donc l'une des représentant.e.s de cette génération des enfants des « disparus », héritière ambigüe d'une histoire aussi tragique qu'inaccessible - les corps de ses parents n'ont toujours pas été retrouvés-, mais aussi dépositaire d'idéaux avortés, impitoyablement réprimés dans le massacre méthodique, systématique, de plusieurs milliers de personnes ${ }^{3}$. Les deux dernières décennies du $\mathrm{XX}^{\mathrm{e}}$ siècle ont été marquées par les conséquences économiques et politiques de ce terrorisme d'État qui a bénéficié à une petite minorité et a entraîné la ruine d'une grande partie du pays ; elles ont abouti à la crise financière et politique de décembre 2001. Selon les déclarations de Daniel Feierstein, chercheur au Conicet et enseignant à l’Université de Buenos Aires :

Le génocide argentin s'est distingué par le niveau d'explicitation, de clarté et d'intentionnalité avec lequel il a proposé la destruction partielle de la population nationale argentine. Et il n'y a pas de meilleure expression pour rendre compte de ce qui est arrivé que celle que les coupables de ce génocide ont eux-mêmes choisie pour baptiser leur entreprise : processus de réorganisation nationale. Cette expression rend compte avec précision de l'objectif et des conséquences de la terreur : transformer la société à travers l'encouragement à la délation, la destruction des réseaux sociaux de solidarité et de coopération, et la création d'un système de méfiance généralisée qui subsiste jusqu'à aujourd'hui, en tant que fondements nécessaires aux transformations économiques et sociales initiées durant la dictature, mais rendues effectives pendant la démocratie, les vingt années qui l'ont suivie (Feierstein, 2011)4.

\footnotetext{
3 Selon les « Mères de la Place de Mai » et les Organisations des Droits Humains, il y aurait plus de 30000 «Desaparecidos». Le célèbre rapport de la commission sur la disparition de personnes (CONADEP) intitulé Nunca más («plus jamais»), coordonné par l'écrivain argentin Ernesto Sábato et publié en 1984 aux Éditions Eudeba, enregistre de la documentation sur 9000 cas. On peut lire ce rapport de la CONADEP sur le site Proyecto Desaparecidos :

http://www.desaparecidos.org/nuncamas/web/investig/articulo/nuncamas/nmas0001.ht $\underline{\mathrm{m}}$ [consulté le 09 avril 2015].

${ }_{4}^{4}$ «El genocidio argentino se destacó por el nivel de explicitación, claridad e intencionalidad con la que se propuso la destrucción parcial del grupo nacional argentino. $\Upsilon$ no hay mejor expresión para dar cuenta de ello que la denominación elegida por los propios genocidas para bautizar su empresa: proceso de reorganización nacional. Esta expresión da cuenta con precisión del objetivo y las consecuencias del terror : transformar a la sociedad a través de la instigación a la delación, la destrucción de las redes sociales de solidaridad y cooperación y la creación de un sistema de desconfianza generalizada que subsiste hasta el presente, como presupuestos necesarios para las transformaciones económico-sociales iniciadas en dictadura pero efectivizadas en la democracia de los veinte años siguientes. » (Feierstein, 2011 - ma traduction).
} 
Le premier film d'Albertina Carri - formée, comme beaucoup de jeunes cinéastes de sa génération, dans l'une des nouvelles écoles de cinéma argentines, la Fundación Universidad del Cine - est un long métrage ambitieux : No quiero volver a casa/fe ne veux pas rentrer à la maison (2000), qui dépeint les tensions d'une société en déliquescence juste avant cette terrible crise de 2001. C'est à travers l'histoire d'un crime dans laquelle se croisent deux familles, l'une de la bourgeoisie industrielle corrompue et l'autre d'une classe moyenne paupérisée, que se construit le portrait d'une ville inhumaine et implacable, aux antipodes des représentations criollistas, folkloristes et convenues, de Buenos Aires. Les choix esthétiques de Carri, noir et blanc, caméra fixe et impersonnelle, narration elliptique à la temporalité inversée (le crime est montré dès la première séquence, son histoire est ensuite représentée par des retours successifs sur le passé), donnent au film un aspect de puzzle à reconstruire dont les images jouent sur une certaine abstraction plastique $^{5}$. Ces options ont suscité à la fois une réelle reconnaissance de la critique et un vif rejet, sur lequel la cinéaste reviendra à plusieurs reprises, car il correspond aux limites assignées à une conception consensuelle du « cinéma féminin » et plus largement de «l'écriture féminine » :

Quand mon film est sorti sur les écrans en Argentine, on m'a accusée de froideur. J'ai lu des commentaires sur mon film du type : «le cinéma doit émouvoir » ou « un film trop froid». Personne n’a osé me dire que ce film, réalisé en noir et blanc, avec une caméra fixe et à peine quelques dialogues, n'était pas digne d'une femme de 25 ans, pourtant c'était un accord tacite. Je doute fort qu'on reproche à un homme son manque d'émotion ou son excès de froideur. D'ailleurs le film ne manquait pas d'émotion, simplement il renvoyait à des états d'âme plus obscurs, rendus silencieux par des expériences très douloureuses, de celles que l'on doit maintenir à distance pour pouvoir les raconter (Carri, 2013).

Los rubios/Les Blonds (2003), son deuxième long-métrage, mêle documentaire et fiction dans un documentaire au second degré où la réalisatrice est présente à l'image, ainsi qu'une actrice : Analía Couceyro,

${ }^{5} \mathrm{~J}$ 'ai proposé une réflexion sur la construction d'une position d'énonciation à partir de ce premier film: «El experimento del rompecabezas. Carri, No quiero volver a casa», El Caleidoscopio autorial. Textualizaciones del cuerpo-corpus : III Congreso Internacional Los Textos del cuerpo, Universidad Autonoma de Barcelona, 2-5 décembre 2014 (en cours de publication). 
chargée de la représenter et qui se présente elle-même au début du film, se nommant et précisant son rôle. Le documentaire inclut les plans du tournage qui demeurent en général hors cadre y compris les discussions et réflexions de l'équipe à propos du refus de financement du projet (Carri, 2007), ou de la difficulté que rencontre Carri au moment d'affronter les émotions que suscitent certains témoignages, les blocages de la mémoire d'une enfant de quatre ans, les médiations convenues qui structurent les témoignages recueillis. Le film intègre par ailleurs des séquences d'animation en stop motion volontairement maladroites de figurines Playmobil qui représentent les parents disparus et les images confuses que peut garder de cette période une enfant de quatre ans, associant innocence bucolique et effroi (Soriano, 2014a). Ce film, extrêmement déstabilisant, l'a rendue célèbre pour la réflexion dérangeante qu'elle y déploie sur les problèmes que pose la représentation des «disparus » et de la mémoire de la dictature militaire, à partir de sa propre histoire familiale et de ses propres souvenirs fragmentaires (Amado, 2004).

En 2005, elle s'unit à Marta Dillon, fille d'une militante « disparue » elle aussi, journaliste et éditrice des suppléments culturels féministe «Las 12 » et LGBTIQ «Soy» du quotidien de gauche argentin Página 12. Dillon va devenir co-scénariste des projets de Carri (Plotkin, 2010).

En 2007, avec l'actrice et metteuse en scène Cristina Banegas, elle réalise un film télévisé intitulé Urgente dans le cadre du cycle national « 200 años » de la chaîne Canal 7 qui célébrait ainsi les 200 ans d'indépendance de la nation Argentine. Ce film est consacré à la question de l'avortement et du viol de mineure et est revendiqué par les réalisatrices comme une réflexion sur « deux cents ans de violence contre les femmes » (Ranzani, 2007).

Dans plusieurs de ses films (longs et courts métrages), Carri mène en outre une réflexion radicale et troublante sur la représentation de la sexualité et sur la pornographie (Bonno, 2014), en particulier dans Barbie también puede eStar triste (court-métrage d'animation, 2001), Géminis (2005), La Rabia (2008) et Pets (2012), un très étrange et très irritant court-métrage pornographique en found footage, qui est conçu comme l'un des éléments d'un 
triptyque composant une installation (Soriano, 2013b) ${ }^{6}$.

Cette même année 2012, dans le pôle opposé de la production audiovisuelle, Carri et Dillon, dont le projet reçut un financement dans le cadre d'un concours national de l'INCAA et du $\mathrm{CDA}^{7}$, ont écrit ensemble une série télévisée en treize épisodes, intitulée 23 pares $^{8}$ (le titre : «23 paires », est une allusion aux vingt-trois paires de chromosomes qui composent le génome humain). L'éventail, très large, du discours de Carri est à considérer comme l'une des données qui caractérisent son positionnement (Mullaly, 2012) et doit être conçu en outre dans le cadre de la politique culturelle menée par le Ministère de la Culture, et en particulier celui de la récente loi sur l'audiovisuel, la «Ley de servicios de comunicación audiovisual», promulguée en 2009. Celle-ci met fin à la loi antérieure imposée par la dictature militaire en 1980. Visant la transparence des critères, la démocratisation de l'espace audiovisuel, le pluralisme, cette loi a suscité un grand débat et son application a été retardée par les recours que le groupe de presse Clarín - dont le monopole a été favorisé par la dictature - a tenté de lui opposer. En automne 2012, toutefois, le gouvernement argentin parvient, après un long bras de fer entre l'État et Clarín (Bullentini, 2012), à faire appliquer la loi considérée, au niveau national et international, comme une loi exemplaire et innovante, orientée par le paradigme des droits humains (Loreti, Charras et Lozano, 2012).

\section{PARES : GENRE EN SÉRIE OU GOMMENT PROPOSER DE NOUVELLES RÉALITÉS}

Rappelons que l'Argentine actuelle constitue un véritable laboratoire dans lequel sont inventés de nouveaux droits et de nouvelles normes de l'humain, à partir des luttes de différents secteurs de la population : celles

\footnotetext{
6 Dans un échange amical de courriers électronique, la réalisatrice expliquait que sa tentative de l'exposer dans un centre d'art contemporain a été différée. Le discours porté par cette œuvre était probablement trop radical. Elle estimait néanmoins qu'il était nécessaire qu'elle travaille à nouveau ce projet.

7 «Instituto Nacional de Cine y Artes Audiovisuales » et «Contenidos Digitales Abiertos » deux entités qui dépendent du Ministère de la Culture.

8 Albertina Carri, 23 pares, série diffusée sur Canal 9, le vendredi à 23h 30, entre le 28 septembre et le 21 décembre 2012.
} 
des militant.e.s de gauche et de leur famille, à la recherche des corps « disparus » de toute une génération massacrée par la dernière dictature militaire (1976-1983); celles des minorités sexuelles systématiquement réprimées par des autorités dont l'idéologie patriarcale, catholique et martiale, est violente et rigide ; enfin celles des femmes qui luttent pour leurs droits (l'avortement n'est pas encore dépénalisé en Argentine et la violence de genre est très élevée ${ }^{9}$ ), mais qui sont également très actives dans les deux secteurs précédents, puisque les «Mères de la place de Mai » ont été à l'initiative des défis contre la dictature militaire et les lesbiennes sont très dynamiques dans les organisations qui intègrent la fédération LGBTIQ. Après la crise économique et politique de 2001 et la recomposition de l'État, le Secrétariat d'État aux Droits Humains a mené une politique beaucoup plus ouverte à la diversité politique et humaine, incluant la reconnaissance des droits des «disparus » et de leur descendance, la récupération de la mémoire des luttes politiques des années 60 et 70, et élaborant un droit à l'identité concernant les enfants des disparus volés à leur famille, mais également attentif à la diversité sexuelle et ethnique, aux discriminations et à la violence de genre. Les lois promulguées ces dernières années, celle dite du «mariage égalitaire » (2010), et celle sur l'« identité de genre » (2012), sont liées à un questionnement plus large sur le droit à l'identité associé à la création de la CONADI (loi 25.457 qui institue la commission nationale pour le droit à l'identité) chargée de veiller à la recherche et à la restitution à leur famille des enfants nés durant la captivité de leur mère que les militaires alors au pouvoir se sont appropriés. L'ensemble de ces mesures découle de la loi créant la Banque Nationale de Données Génétiques (1987) et la loi d'abrogation de la «Ley de Punto final » et de la «Ley de obediencia debida », de 1998, qui rend à nouveau possibles les poursuites à l'encontre des militaires qui ont commis des crimes pendant la dictature ${ }^{10}$.

9 On peut consulter les documents disponibles sur (entre autres) : http://www.csjn.gov.ar/om/docs/femicidios_2014.pdf ; http://www.onu.org.ar/la-cortesuprema-de-justicia-de-la-nacion-presento-el-primer-registro-nacional-de-femicidios-de-lajusticia-argentina/ ; $\quad$ http://www.abortolegal.com.ar/?tag=mortalidad-materna ; http://www.lanacion.com.ar/1396232-las-cifras-del-aborto-clandestino-en-el-pais [consultés le 09 avril 2015].

10 On lira l'ensemble des mesures adoptées depuis une dizaine d'années: conventions 
La série télévisée prend en charge deux des questions clés qui hantent les débats nationaux sur les droits humains (Soriano, 2014c). D’une part, un débat sur l'archive inaccessible, sur l'héritage, ravi et détruit par la normalisation autoritaire de la dictature militaire, des milliers de « disparus » et des centaines d'enfants volés, que les bourreaux de leur mère se sont appropriés, privés du droit de connaître leur histoire et de réclamer le châtiment des tortionnaires. D'autre part, un débat sur la violence de genre, le viol, l’inceste, le féminicide, l'avortement, l'intersexe, l'homosexualité, la diversité sexuelle, qui concerne des milliers de personnes privées de droits au nom d'une mystique hétérosexuelle dont les normalisations violentes, quelquefois fatales, sont maintenues invisibles. En transposant ce double débat dans les trames qui régissent les épisodes de 23 pares, Albertina Carri en assume les termes, et engage à la fois un nouveau questionnement, qui concerne les territoires du visible, les stratégies d'invention de formes de représentation, de savoirs inédits et de territoires culturels à venir, à conquérir.

À l'opposé des positions conservatrices du naturalisme déterministe qui souvent se fondent sur une conception figée de la biologie - la patrimonialisation en jeu dans ce motif récurrent du génome, qui donne son titre à la série TV, entraîne la biologie moléculaire dans une conception constructiviste et politique de l'identité, qui procède de cet «indice de abuelidad» (que l'on pourrait traduire par «indice de grand-maternité », autrement dit marque génétique de filiation de seconde génération). Il s'agit là d'une innovation scientifique qu'ont obtenue des généticiens auxquels elles se sont adressées, grâce à leur quête inlassable, les Abuelas : les « Grandmères » de la place de Mai (Abuelas Plaza de Mayo 2008). C'est cette dimension politique et culturelle de la filiation et de l'identité que Carri et Dillon exposent dans les 13 épisodes de la série télévisée qui s'articule autour des cas traités par un laboratoire d'analyses génétiques, à partir de la métonymie des « 23 paires ». Le motif du génome renvoie donc à ces luttes 
des Mères et Grand-mères de la Place de Mai, à leurs recherches et revendications, et aux travaux de l'Équipe Argentine d'Anthropologie Légale $\left(\mathrm{EAAF}^{11}\right)$ qui, grâce à la Banque Nationale de Données Génétiques, s'emploient à reconstruire l'histoire des disparus, en identifiant les dépouilles et leurs descendant.e.s. Le génome devient par conséquent, dans cette série, l'un des symboles de la complexité des politiques de l'identité dont la réalisatrice se propose de témoigner.

Sur le plan formel, nous observons dans 23 pares l'une des caractéristiques majeures des réalisations de Carri : le mélange des genres, détonnant, qui produit une défamiliarisation peu fréquente dans les séries télévisées, et qu'elle revendique pour l'ensemble de sa production dans les entretiens qu'elle concède à la presse. À ce mélange s'ajoutent des procédés récurrents de distanciation parodique. Au drame de la reconnaissance, motif clé du mélodrame, qui s'étire sur l'ensemble des épisodes au niveau de la famille des protagonistes, mais qui caractérise également un certain nombre de récits seconds, s'ajoutent les ressorts de l'intrigue policière, la figure du profiler et les miracles de l'expertise scientifique, mais aussi la parodie du film d'horreur («Le mort qui parle »), l'érotisme, la fiction documentaire consacrée à l'inceste et au droit à l'avortement (« Le droit de décider »), à la discrimination hétéronormative («Famille Royale»). Alors que le mélodrame est l'une des formes narratives qui naturalisent le système sexe / genre (Rubin, 1975) - même s'il s'emploie à dénoncer un ordre injuste imposé par une figure malfaisante - les réécritures distanciées de ce genre que proposent Dillon et Carri dans 23 pares, ont pour résultat deux déplacements fondamentaux. D’une part, la transgression des normes qui ouvre l'intrigue est neutralisée, car ces normes sont ramenées à une normativité arbitraire, issues de rapports socioculturels injustes et discriminatoires. Elles sont ainsi mises à l'épreuve de normes nouvelles, historicisées. D'autre part, l'enjeu du nœud dramatique n'est plus le triomphe d'un Bien convenu, il devient la tentative d'invention de nouvelles normes de l'humain, plus ouvertes, progressivement naturalisées, et la

${ }^{11}$ On consultera leur site web pour plus d'information : http://www.eaaf.org. 
démonstration de la capacité d'agir des personnages, qui échappent ainsi aux constructions stéréotypées qu'utilisent ordinairement les séries.

Revenons sur le mélodrame familial, forme que Carri utilise sur un mode parodique dans sa production (Soriano, 2013a) et qui court sur l'ensemble de la série. Le motif du secret est l'un des traits majeurs du mélodrame, car les péripéties s'articulent sur la tension entre la répression du message et la nécessité de reconnaissance de celui-ci (Brooks, 1995 : 28). La structure hermétique procède de la censure et de la désinformation instaurées par le régime autoritaire, qui engendrent à leur tour des discours chiffrés, néanmoins elle peut être également rapprochée de la critique de la « propagande » médiatique que dénonce Carri dans sa conférence. Dans 23 pares le motif du génome est au cœur de cette tension, il fonctionne comme une matrice à la fois secrète, inaccessible et cependant omniprésente dans les déterminations qu'il engendre. Cette problématique cruciale du cryptage qui structure la série implique que les deux états d'un message entrent dans une logique spéculaire qui suspend tout recours à une supposée vérité originelle : ne demeurent que des versions possibles de la représentation et leurs effets contradictoires, parmi lesquels il nous revient de nous orienter. L'ambivalence parodique engendre un rapport complexe, fait de reconnaissance des codes et de distanciation, qui exige du public une incessante prise de position face aux valeurs contestées dans l'intrigue. La compréhension «littérale » n'est pas impossible, mais elle est brouillée, d'infimes décalages déclenchent une seconde lecture, critique, dont le sens ne peut être atteint que sur le mode d'un savoir situé.

Le commentaire de l'une des deux protagonistes principales : «mi vida se parece a un culebrón mexicano» («ma vie ressemble à un mauvais feuilleton mexicain »), introduit un niveau supplémentaire de spécularité. Dans ces propos parodiques, la hiérarchie nationaliste ironiquement suggérée entre la «bonne» série argentine et la «mauvaise » série mexicaine - le Mexique est, avec le Venezuela, l'un des principaux producteurs de séries télévisées dans l'Amérique hispanophone- est anecdotique, elle fait partie des codes du vraisemblable; il convient de considérer que dans cette mise en abyme détournée, c'est l'écart entre les 
codes qui régissent 23 pares et ceux qu'impose le standard du feuilleton télévisé qui s'exhibe. Il se situe principalement dans les normes en jeu dans les différents récits et indique la présence d'un discours féministe radical et queer qui prend en charge les questions du débat contemporain sur les droits humains. Celles-ci, dans le cas de l'Argentine, sont issues de l'éclatement historique de la frontière entre la vie privée et l'espace public qu'ont opéré les Mères et les Grand-mères de la Place de Mai. Ces femmes argentines, dont les co-scénaristes Carri et Dillon sont les «filles », ont révélé et mis en œuvre le potentiel révolutionnaire de ce qui fut l'un des objectifs fondamentaux de la deuxième vague du féminisme: comprendre et revendiquer la dimension politique de l'intime; elles sont devenues ainsi actrices de leur histoire et actrices principales de l'histoire de l'Argentine contemporaine.

C'est donc aussi un patrimoine politique et culturel longtemps censuré, ou détruit, que cette génération des enfants de disparus revendique aujourd'hui, et dans le cas de Carri un patrimoine cinématographique. Rappelons que le cinéma des années 60-70 en Argentine, comme dans d'autres pays d'Amérique latine, s'est défini avant tout comme un cinéma politique et populaire, refusant à la fois la recherche esthétique élitiste du cinéma d'auteur européen, et l'inaccessible modèle hollywoodien, dont les moyens étaient inconcevables dans le contexte économique des pays d'Amérique latine ${ }^{12}$. L'incessante recherche formelle et la production militante, artisanale, étaient alors pensées ensemble, en tant qu'arme politique. La priorité donnée à l'accessibilité du discours ne supposait en aucun cas le renoncement au travail d'élaboration formelle, bien au contraire, puisque la production de nouvelles connaissances était en jeu et qu'elle exigeait le questionnement des codes et des catégories. Albertina Carri s’inscrit dans cette généalogie à laquelle elle rend hommage dans son court métrage Restos de 2010 (Soriano, 2014a).

12 On lira à ce sujet les témoignages et documents dans : VVAA, Hojas de cine. Testimonios y documentos del nuevo cine latinoamericano (1988), Volumen 1. Centro y Sudamérica. México, Secretaría de Educación Pública, Universidad Autónoma, Fundación mexicana de cineastas. 
Avant d'aborder le téléfilm Urgente, je voudrais compléter cette réflexion sur ce positionnement esthético-politique. Pendant l'année 20132014, Carri s'est engagée dans une nouvelle entreprise militante: sur une proposition du Secrétariat d'État aux Droits Humains, elle fut la directrice artistique du premier festival international de films LGBTIQ de Buenos Aires, Asterisco, qui a eu lieu du 3 au 8 juin 2014. Dans le Journal du festival, qu'elle a écrit pendant la préparation, on peut lire :

Je crois en la forme et je soutiens, avec mon corps et ma façon de vivre, et avec tout ce qui pourra être nécessaire, que la politique est forme, et je ne vais pas choisir des films pour leur thématique, ni remplir les cases pour chaque lettre, si les films ne correspondent pas à un modèle narratif de rupture du sens commun, au-delà du sujet qu'ils racontent (Carri, 20132014, ma traduction).

La phrase citée renvoie à l'absence, déplorée par Carri, de film sur l'intersexualité dans son festival LGBTIQ: elle n'a pas pu remplir la case $I$. Or deux réalisatrices argentines ont chacune consacré un long-métrage à des personnages intersexes : Lucía Puenzo, XXY (2007) et Julia Solomonoff, El último verano de la boyita (2009). Ces deux films, qui ont eu pourtant un fort retentissement, national et international, ne correspondent donc pas aux critères qu'énonce Carri. Au-delà de la prise de position que ce choix manifeste - dans un champ cinématographique national très politisé, et dans un champ politique très tendu - que pouvons-nous lire dans ce rejet? Un geste autoritaire et élitiste? Ou une exigence politique, à la fois esthétique et épistémologique, qui dénonce les violences invisibles de la normalisation? Pour ma part, je pencherais pour la deuxième interprétation, compte tenu du contexte brièvement évoqué. Mais la question reste ouverte et les réponses soumises à une analyse précise des stratégies adoptées par chacun de ces deux films, qui dénoncent la violence de la normalisation hétérosexuelle au moyen de récits dont la «normalité » mimétique pourrait nous paraître « excessive ».

\section{URGENTE : MODERNITÉS AUDIO-VISUELLES ET ARGHAÏSME PATRIARGAL}

Dans les limites de cette réflexion, je n’analyserai que quelques 
aspects du téléfilm intitulé Urgente, afin d'explorer d'autres fonctionnements des dispositifs esthétiques de distanciation critique que crée Albertina Carri. En premier lieu, notons qu'il s'agit une nouvelle fois d'un film hybride, qui inclut et surtout entrechoque différents genres et différentes pratiques: télévisuelles, théâtrales et cinématographiques. Il est co-dirigé par Albertina Carri et Cristina Banegas, dans le cadre du programme «200 años» de la chaîne nationale Canal 7 qui, à l'occasion des célébrations du bicentenaire de l'indépendance, a organisé une série de collaborations entre cinéastes et metteurs ou metteuses en scène de théâtre (Gasparín, 2012; Ranzani, 2007 ; Pérez Llahí, 2008). L’hybridité est donc issue en partie du projet luimême (Gasparín, 2012), cependant elle est réappropriée de façon hyperbolique par Carri dans un traitement de l'espace et des points de vue extrêmement dérangeant.

La dimension documentaire procède du point de départ du téléfilm : un fait divers récent qui obsède la réalisatrice au moment de la conception du projet (Ranzani, 2007) : une fillette de onze ans s'est suicidée à Jujuy, dans les toilettes de son école, parce qu'elle était enceinte. Cependant Carri et Banegas n'adoptent pas la rhétorique réaliste des documentaires de presse, elles construisent un dispositif scénographique qui crée la continuitécontiguïté des espaces représentés - un dispositif que Carri compare à celui du film de Lars Von Trier en qualifiant Urgente de «Dogville criollo» (Ranzani, 2007) - et mettent ainsi en scène l'implacable clôture socioidéologique et la complicité des différentes institutions, dont le silence condamne à mort une adolescente violée et enceinte: la famille, l'école, l'Église. Le film exploite la tension entre, d'une part, la proximité et la banalité du fait divers, qui exhibe, mais en même temps occulte un contexte national extrêmement violent, et d'autre part le dispositif scénographique qui, dans un immense plateau de télévision au sol recouvert de terre rouge, trace au moyen d'accessoires minimalistes à valeur métonymique les espaces stratégiques du hameau isolé : l'école, l'église et la cure, la maison familiale de la matrone du village, grand-mère de l'adolescente violée, qui pratique des accouchements et des soins traditionnels et vit seule avec sa fille et sa petite fille. 
Deux événements viennent troubler la paix bucolique et animent les conversations: l'énigme du mutisme et des malaises de la jeune fille et l'arrivée très attendue de la télévision dans cette communauté isolée d'une zone reculée de Misiones, une province du Nord-Est argentin, très rurale, occupée par la forêt tropicale, et où le dynamisme social est très faible (les femmes ne sont pas intégrées au marché du travail et les jeunes gens, peu formés, en sont exclus également $\left.{ }^{13}\right)$. Le contraste de ces deux événements est exploité et monte en puissance jusqu'à la fin du film; la modernité désirable est matérialisée par l'un de ses fétiches paradigmatiques: la télévision, alors que l'archaïsme honteux est assumé par le corps souillé de la jeune fille, victime émissaire sommée de disparaître pour que l'heureux événement puisse voir le jour. La dichotomie "Civilisation vs barbarie » qu'a élaborée au XIX $\mathrm{X}^{\mathrm{e}}$ siècle l'un des plus grands écrivains argentins, essayiste et homme d'État, Domingo Faustino Sarmiento (Sarmiento, 1945), pour justifier son projet politique de modernisation libérale, et qui depuis fonctionne dans les discours qui racialisent la population pauvre et opprimée, se profile dans la machinerie sociale mise en scène et que le film de Carri va dénoncer.

Dans cet espace sans issue qui la cerne, la jeune fille n'est jamais nommée, désignée comme «la Nena » (la Fille) elle n'est identifiée que grâce à son genre et à sa position dans la généalogie familiale. Les autres personnages sont eux aussi désignés par leur fonction sociale ou familiale : « la Maîtresse d'école », « le Curé », « la Matrone » qui est aussi la « Mère » et la «Grand-mère », etc. Les identités génériques soulignent les rapports sociaux dans lesquels sont insérés les personnages. Ces dénominations déjouent la rhétorique médiatique qui traite ce type de situation suivant le modèle réaliste du «fait divers » (Houel, Mercader et Sobota, 2003), et joue avec la typologie théâtrale, fortement politisée dans la production contemporaine argentine, dont la dramaturge Griselda Gambaro est l'une des figures majeures. La rupture des codes de représentation est nécessaire

${ }^{13}$ Gustavo Braier, Norma Esper, Liliana Corinaldesi « Estudio de tendencias y perspectivas del sector forestal en América latina al año 2020. Informe nacional complementario. Argentina », Depósito de documentos de la FAO, Food and Agriculture Organisation of the United Nation, http://www.fao.org/docrep/006/j2053s/j2053s12.htm [consulté le 9 avril 2015]. 
pour rendre compte de la réalité structurelle de la violence de genre (Femenías 2004, 2008 et 2014), inter-dite, invisible ou érotisée dans les représentations normalisées par les codes masculinistes (Soriano, 2008 et 2009) qui diffusent les valeurs patriarcales assumées par l'Église et l'École. Mais l'issue à la situation de l'enfant est également - et fatalement - différée par les débats au sein de sa famille, où s'affrontent trois femmes qui incarnent les positions en tension dans le débat national sur l'avortement (Sciortini, 2014): la grand-mère, qui préconise le recours aux techniques ancestrales ; la tante de la jeune fille, sage-femme à l'hôpital, qui voudrait, pour sa sécurité, l'amener dans son service, prenant ainsi le risque de la livrer aux sanctions de la loi ; et enfin la mère, jeune mère célibataire ellemême, qui pense que sa fille devrait à son tour, comme elle le fit, garder l'enfant à naître.

La «Nena » est inter-dite, on la dit: elle est l'objet de tous les discours, des querelles et des alliances que déclenchent son «état», aussi étouffé que son histoire. Elle est devenue mutique (le personnage ne participe à aucun échange), car elle est condamnée au silence, à un mutisme absolu. La celation de sa grossesse n'est rompue que par un dédoublement du dispositif d'énonciation qui permet de déjouer la nomophatique et l'intimidation cognitive (Soriano, 2014b ; Mullaly et Soriano, 2014). Dans le téléfilm, cette mise en abyme ouvre une issue et accueille la parole, les angoisses et le désespoir de l'enfant, dans un espace onirique où sa voix se fait entendre. La scénographie de cet espace alternatif est complexe, multiple, le discours est médiatisé : la jeune fille est assise à son pupitre d'écolière et écrit une lettre à ses sœurs. Sa voix, en voix off, énonce ce qu'elle est en train d'écrire, mais l'actrice elle-même demeure muette. Devant elle, sur un écran noir, sont projetées des silhouettes géométriques et géantes de chiens bleus qui s'agitent frénétiquement, alors que la bande son est saturée de halètements et de grognements sonores. À sa droite, plus bas, une télévision montre des scènes d'accouchements de différents animaux. Trois niveaux d'images, quatre niveaux sonores : la voix off, les grognements et halètements des chiens, un grondement sourd continu et des cris de bébé à la fin de la séquence, qu'interrompt le claquement d'une explosion au 
moment du réveil brutal de la jeune fille. Cet espace onirique est la scène seconde de l'art, de l'artifice, celle du cinéma tel que le définit Carri dans sa conférence, autrement dit celle qu'assume le téléfilm en tant que dispositif technologique et sémiotique.

Cette séquence qui exhibe le monstrueux cauchemar de la jeune fille, enceinte, torturée par ce corps étranger qui pousse dans son corps, au moment où elle annonce à ses sœurs, destinataires de sa lettre, qu'elle va mourir, est caractérisée par un angle de prise de vue doublement intéressant : la caméra est située derrière le personnage, mais à une hauteur humaine. Or, outre la scénographie du type Dogville, l'un des procédés de défamiliarisation qui caractérisent ce film est la récurrence des prises de vues en plongée. La caméra, souvent située à moins d'un mètre du sol, se centre sur les pieds des personnages et accompagne leurs parcours sur la terre rouge. Ces cadrages écrasent, voire anéantissent la perspective et disent l'accablement, l'oppression subie, comme s'ils représentaient un regard rivé au sol. L'horizon est absent et son absence est encore redoublée par les tentures sur lesquelles sont projetées des images conventionnelles (arbre, soleil, ciel crépusculaire) qui oblitèrent la perception de l'espace et bornent la représentation avec des clichés. Ces cadrages alternent avec des prises de vue en plongée zénithale où la caméra est en position haute, ou très haute : de trois à cinq mètres du sol. Les personnages apparaissent ainsi écrasés, leurs formes sont presque inhumaines et peu reconnaissables, après coup, ils paraissent animalisés et comme enfermés dans la boîte que constitue le plateau. Ces angles de prise de vue produisent de puissants effets de distanciation et de défamiliarisation, en rompant avec la rhétorique consensuelle qui mime un regard anthropomorphe, à hauteur d'homme; ce dispositif complexe, en anamorphose (Soriano, 2014b ; Mullaly et Soriano, 2014), révèle l'artificialité et l'irrecevabilité d'un point de vue neutre. Ce dernier apparaît alors dans sa réalité de construction rendue invisible par sa fréquence, liée à l'usage conventionnel de codes de représentation normalisés.

À la question posée par Oscar Ranzani, critique de cinéma, sur la possibilité de provoquer un débat sur l'avortement à partir d'une fiction, lors 
d'une interview consacrée à Urgente, Carri répond :

L'art te donne la possibilité de penser les choses d'une autre façon et dans d'autres termes. Et il te frappe aux endroits les plus intimes par moment, ce n'est pas l'histoire d'un autre, cela peut devenir quelque chose qui serait un peu plus universel. Ge n'est plus un cas particulier, cela devient un événement quotidien avec lequel chacun peut s’identifier (Ranzani, 2007, ma traduction).

Carri revendique donc pour l'art ce que Françoise Collin exigeait des révélations assumées par la critique féministe : une création « comme œuvre à signification universalisable ». Le viol et la dépénalisation de l'avortement sont des questions rendues universelles par les modalités esthétiques de leur traitement. Le patriarcat et son hypocrisie tuent les femmes, celles qui sont les plus vulnérables: les femmes des milieux aisés ne rencontrent aucune difficulté pour avorter. Les jeunes femmes, les femmes pauvres, celles qui sont enfermées dans l'ignorance et la rigidité des traditions, sexualisées et racialisées par les discours de l'Église et de l'École sont les victimes quotidiennes de la violence de ces institutions. En effet, leurs représentants dans Urgente prétendent que la situation de la Nena est une fatalité : «c'est de famille, c'est dans le sang, elles aiment ça, elles ne comprennent même pas ce qu'est le péché »; autrement dit c'est un problème sans issue qui concerne les «autres », et non la société normale. Or l'impact des derniers plans du film nous frappe dans notre intimité et nous bouleverse.

Un long plan séquence, un travelling en plongée, montre la Nena qui se lève de son lit, pendant la nuit, puis montre seulement son parcours dans le village : elle demeure désormais hors champ. Tous les autres personnages sont montrés successivement dans leur sommeil : personne ne veille sur l'enfant qui s'éloigne ; ce plan fait écho au premier plan du film, dans lequel la jeune fille est représentée circulant seule dans la nuit. La caméra prend ensuite de la hauteur, tout le village est montré dans son ensemble en plongée, alors que retentit un cri enthousiaste : «La télévision est arrivée ! La télévision est arrivée!». Immédiatement après, en plans rapprochés « excessivement normaux», les habitants sont montrés les uns après les autres, qui se rassemblent à la sortie du hameau, face au soleil levant qui les 
nimbe de lumière, pour accueillir l'obscur objet de leur désir unanime. Cet objet - la télévision - demeure absent, mais omniprésent à la fois, en tant que point vers lequel convergent tous les regards des personnages qui l'attendent avec impatience, représentés de dos, en plan d'ensemble. Un long et lent travelling arrière et vers le haut éloigne ensuite la caméra jusqu'à faire entrer dans le cadre, par la droite, au premier plan, une image que le spectateur et la spectatrice anticipent et redoutent: les pieds de la jeune fille qui s'est pendue, et qui se balancent doucement. En arrière-plan, en bas du cadre, qui lui tournent définitivement le dos, les habitants du village rassemblés continuent d'attendre la télévision...

... Mais il y a aussi le cinéma. Nous pouvons refuser de partager les catégories proposées par Carri, les nuancer, les complexifier. Il me semble cependant intéressant d'explorer les différents aspects de son engagement en faveur de formes qui s'écartent d'une mimesis complaisante et consensuelle. Son avant-gardisme, qui s’inscrit dans une généalogie militante, rompt avec une mimesis qui opère la normalisation et la naturalisation de l'injustice et des violences sociales, mais déjoue par ailleurs les cadres d'un hermétisme élitiste. Le défi que relève Carri est assez comparable à celui que Collin décrit : une négociation incessante et risquée entre le visible et l'invisible, le dicible et l'interdit, les idées reçues et l'irrecevable, une négociation qui construit, jour après jour, une sous-culture minoritaire féministe queer qui tend à élargir les normes de l'universel, car la culture est un espace où se jouent les luttes symboliques qui sont à la fois des luttes politiques. Les propos qu'elle diffuse sur le journal du festival de cinéma LGBTIQ dont elle a assumé la programmation, éclaireront mes hypothèses de lecture :

Astérisco n'est pas seulement un festival, c'est une forme d'être au monde, une réunion d'ami*s, une manière de célébrer le chemin parcouru et de penser à la façon dont nous pouvons continuer le changement culturel $[\ldots]$.

Cinéma d'horreur, postporno, documentaires de dénonciation et fictions exhumées forment une trame, une réalité qui détruit les ordres qui oppriment parfois au moyen d'une violence textuelle, parfois en allant jusqu'à une violence assassine ; cet énorme film de cent cinquante heures qu'est Asterisco aidera, j'en suis sûre, à penser de nouveaux paradigmes de sensualité et de désir, de discussion et de vie partagée (Carri 2013-2014, 
ma traduction).

Le cinéma que revendique Carri ressemble assez à celui qu'anticipait Virginia Woolf en 1926: «Quelque chose d'abstrait, quelque chose qui évolue avec un art conscient et contrôlé, quelque chose qui demande aux mots ou à la musique un peu d'aide pour se rendre intelligible, juste en les bouleversant - de tous ces mouvements et de ces abstractions, les films seront composés dans l'avenir » (Woolf, 2012: 17). Un art en mouvement, un art du mouvement, contre toutes les formes de fixité naturaliste, un art de la défamiliarisation plastique et cognitive : un cinéma féministe.

\section{BIBLIOGRAPHIE}

AbUelaS DE PLAZA DE MAYO (éd.) (2008), Las abuelas y la genética. El aporte de la ciencia en la búsqueda de los chicos desaparecidos, "Identidad, Familia, Libertad», Abuelas de Plaza de Mayo [en ligne] http://www.abuelas.org.ar/material/libros/LibroGenetica.pdf [consulté le 09 avril 2015].

AmAdo Ana (2004), « Órdenes de la memoria y desórdenes de la ficción », dans Ana Amado et Nora Dominguez (dir.), Lazos de familia. Herencias, cuerpos, ficciones, Buenos Aires, Paidós, p. 45-109.

Bonno Justine (2014), «Poupées pornos, violence ethnographique et patrimoine de la domestication: reconnaissance du genre postpornographique dans la production de la réalisatrice Albertina Carri », dans Laurence Mullaly et Michèle Soriano (dir.), De cierta manera : cine y género en América latina, Paris, L'Harmattan, collection Sexualité et genre, p. 169-191.

Brooks Peter (1995), The melodramatic Imagination, New Haven \& London, Yale University Press.

CARRI Albertina (2014), «Sobre cuerpo y género en el audiovisual argentino », dans Laurence Mullaly et Michèle SoRiano (dir.), De cierta manera: cine y género en América latina, Paris, L'Harmattan, collection Sexualité et genre, p. 243-251.

CARRI Albertina (13.08.2013-26.05.2014), «Diario del festival de cine LGBTIQ. Putos hubo siempre », Crónica, Revista Anfibia, Universidad Nacional San Martín. [en ligne] http://revistaanfibia.com/nueva/cronica/putos_hubo_siempre/ [consulté le 09 avril 2015].

CARRI Albertina (2013), «Cuestión de imagen », Cinémas d’Amérique latine, 
Toulouse, Presses Universitaires du Mirail, n² 21, p. 30-41.

Collin Françoise et Irène KaUfer (2014), Parcours féministe, DonnemarieDontilly, Éditions iXe.

COLLIN Françoise et Irène KAUfER (2014), «L'empire des signes », dans Françoise Collin et Irène KaUfer, Parcours féministe, DonnemarieDontilly, Éditions iXe, p. 135-157.

Femenías María Luisa (2014), «Penser et montrer la violence. Catégories et modalités ", Caravelle, "Citoyenneté et formes de violence. La violence de genre en Amérique latine », Toulouse, Presses Universitaires du Mirail, n 102, p. 21-36.

Femenías María Luisa (2008), «Violencia contra las mujeres : urdimbres que marcan la trama », dans Elida APONTE SANCHEZ et Maria Luisa FEMEnías (dir.), Articulaciones sobre la violencia contra las mujeres, La Plata, Editorial de la Universidad de La Plata, collection Campo Social, p. 12-53.

Femenías María Luisa (2006), «Afirmación identitaria, localización y feminismo mestizo », dans María Luisa Femenías (dir.), Feminismos de París a La Plata, Buenos Aires, Catálogos, p. 97-125.

Femenías María Luisa (2004), «Lectura excéntrica y cambio de paradigma : des-invisibilización de los a priori históricos de género », Imprévue, «Théories critiques et littérature latino-américaine actuelle », Montpellier, Éditions du CERS, n 1-2, p. 207-225.

Feierstein Daniel (2011), «Proceso de Reorganización Nacional », Página 12, 24.03.2011 [en ligne] http://www.pagina12.com.ar/diario/elpais/subnotas/16480652714-2011-03-24.html [consulté le 09 avril 2015].

Gaussot Ludovic (2005), «Des rapports sociaux de sexe à la connaissance de ces rapports : une vertu cognitive de la non-conformité ? ", Cahiers du Genre, no 39, p. 153-172. DOI : 10.3917/cdge.039.0153.

Houel Annick, Patricia Mercader et Helga Sobota (2003), Crime passionnel, crime ordinaire?, Paris, Presses Universitaires de France, collection Sociologie d'aujourd'hui.

IRIBARREN María (2008), "'Sufro con el exceso de normalidad' entrevista a Albertina Carri », Cinecrópolis [en ligne] http://www.cinecropolis.com/entrevistas/albertinacarri.htm [consulté le 09 avril 2015].

Le Doeuff, Michèle (1998), Le Sexe du savoir, Paris, Aubier.

LORETi Damián, Diego DE Charras et Luis Lozano (2012), «Comunicación audiovisual, todo lo que hay que saber », Página 12, 24.10.2012 [en ligne] http://www.pagina12.com.ar/diario/elpais/1206213-2012-10-24.html [consulté le 3 janvier 2014].

Mullaly Laurence (2012), "Albertina Carri. Cinéaste de l'inconfort ", Cinémas d'Amérique Latine, Toulouse, Presses Universitaires du Mirail, $\mathrm{n}^{\circ} 20$, p. 151-162. 
Mullaly Laurence (2014), «El no de las niñas: la violencia de género según Albertina Carri », dans Laurence MulLaLY et Michèle SORIANO (dir.), De cierta manera : cine y género en América latina, Paris, L'Harmattan, collection Sexualité et genre, p. 219-239.

Mullaly Laurence et Michèle Soriano (2014), «Introducción. De cierta manera: cuando las cineastas latinoamericanas reconfiguran las normas de género », dans Laurence Mullaly et Michèle SORIANO (dir.), De cierta manera : cine y género en América latina, Paris, L'Harmattan, collection Sexualité et genre, p. 9-24.

NAudier Delphine et Brigitte ROLLET (2007), «Introduction », dans Delphine NAUDiER et Brigitte RolLET (dir.), Genre et légitimité culturelle. Quelle reconnaissance pour les femmes?, Paris, L'Harmattan, collection Bibliothèque du Féminisme, p. 9-20.

Plotkin Pablo (2010), "Albertina Carri y Marta Dillon : retrato de una nueva familia », Rolling Stones, 12.08.2010 [en ligne] http://www.rollingstone.com.ar/1294010 [consulté le 27 avril 2015].

Ranzani Oscar (2007), «El aborto, en una mirada Urgente », Página 12, 26.06.2007 [en ligne] http://www.pagina12.com.ar/diario/suplementos/espectaculos/86761-2007-06-26.html [consulté le 09 mars 2015].

RubiN Gayle (1975), "The Traffic in Women: Notes on the Political Economy of Sex », Toward an Anthropology of Women, New York, Monthly Review Press, p. 157-210. Traduction française: Gayle RuBIN (2010), Surveiller et jouir. Anthropologie politique du sexe, Paris, EPEL.

SARMiento Domingo Faustino (2000 [1945]), Facundo. Civilización y Barbarie. Vida de Juan Facundo Quiroga, édition de Roberto YAHNI, Madrid, Cátedra, collection « Letras hispánicas ».

Sofio Séverine, Perin Emel Yavuz et Pascale Molinier (2007), «Genre, Féminisme et valeur de l'art», Cahiers $d u$ Genre, nº 43, Paris, L'Harmattan.

SORIANO Michèle (2008), «Violence, érotisme, pornographie : technologies du genre dans les genres policier et érotique », Lectures du genre, «Lectures théoriques, approches de la fiction » $\mathrm{n}^{\circ 5}$ [en ligne] http://lecturesdugenre.fr/lectures_du_genre_5/Soriano.html [consulté le 09 avril 2015].

SORIANO Michèle (2009), « Rendre visible l'indicible frontière : des interdits à l'interdit du discours ", Colloque "Interdit E̊ genre ", 15-16 mai 2009, MSH - Université François Rabelais, Tours.

SORIANO Michèle (2013a), «De la niña inútil a la Barbie pornostar: melodrama, género y canon en el discurso feminista latinoamericano », dans María A. Semilla Duran (dir.), Variaciones sobre el melodrama, Madrid, Casa de Cartón, p. 397-424.

SORIANO Michèle (2013b), «Représenter la sexualité, repenser le sexe : Pets (2012) d'Albertina Carri », Lineas, $\mathrm{n}^{\circ} 3$, «Les paradigmes Masculin/Féminin sont-ils encore utiles?» Partie 2 Études 
analytiques [en ligne] http://revues.univ-pau.fr/lineas/1101.

SORIANO Michèle (2014a), «Animación: distanciar, desnaturalizar, experimentar. Dispositivos híbridos de Albertina Carri», dans Laurence Mullaly et Michèle Soriano (dir.), De cierta manera : cine y género en América latina, Paris, L'Harmattan, collection Sexualité et genre, p. 193-218.

SORIANO Michèle (2014b), « Cuando se juega el cuerpo. Saberes situados y nomofática », dans Marie Agnès PALAISI-ROBERT et Meri TORRAS (coord.), El cuerpo en juego: cartografia conceptual y representaciones en las producciones culturales de América Latina, Paris, Mare et Martin, collection LLAMA, p. 57-76.

SORIANO Michèle (2014c), «23 pares, d'Albertina Carri. De l'intime à l'histoire ", Transmissions textuelles, dans Jeanne RAIMOND et JeanLouis BRUNEL (dir.), Cahiers du GRES, Institut International de Sociocritique, Université de Nîmes.

WOOLF Virginia (2012), Le cinéma et autres essais, Paris, Les Éditions de Paris - Max Chaleil.

\section{CORPUS}

CARri Albertina, Cristina Banegas (2007), Urgente, Canal 7 (production), Argentina, Telefilm $76 \mathrm{mn}$.

CARRI Albertina (2012), 23 pares, série diffusée sur Canal 9, le vendredi à 23 h 30, entre le 28 septembre et le 21 décembre 2012.

CARri Albertina (2008), La Rabia, Matanza cine, Pablo Trapero et Albertina Carri (production), Argentina, $83 \mathrm{mn}$, couleur.

CARri Albertina (2007), Los Rubios. Cartografia de una película, Buenos Aires, Ediciones Gráficas especiales.

CARRI Albertina (2003), Los Rubios, Albertina Carri et Barry Ellsworth (production), Argentina, $89 \mathrm{mn}, 35 \mathrm{~mm}, \mathrm{~B} \& \mathrm{~N} /$ couleur.

CARRI Albertina (2001), Barbie también puede eStar triste. Melodrama pornográfico, Argentina, $24 \mathrm{mn}, 35 \mathrm{~mm}$, couleur.

CARRI Albertina (2000), No quiero volver a casa, Albertina Carri (production), Argentina, $74 \mathrm{mn}, 35 \mathrm{~mm}, \mathrm{~B} \& \mathrm{~N}$. 